\title{
Neural network principles for theoretical psychology
}

\author{
DANIEL S. LEVINE \\ University of Texas at Arlington, Arlington, Texas
}

\begin{abstract}
Neural networks are an increasingly important tool for the mechanistic understanding of psychological phenomena. Three commonly used principles in neural-network design (associative learning, competition, and opponent processing) are outlined here, and two examples of their use in behavior-modeling architectures are discussed. One example relates to an instance of reinforcement learning; that is, of an organism controlling its environment to maximize positive reinforcement or to minimize negative reinforcement. The other example relates to some characteristic deviations from reinforcement learning that occur in people or monkeys with frontal-lobe damage.
\end{abstract}

Neural network research has gone from rags to riches in less than a decade. The popular press has followed this trend, with articles in Newsweek, The New York Times, The Wall Street Journal, and elsewhere (see, e.g., Stipp, 1988). However, most of these articles have not done justice to the significance of neural-network models for psychology. For the first time in history, experimental (and clinical) psychology can draw on a partnership with theoretical psychology, much as experimental and theoretical physics have coexisted symbiotically since the early $1900 \mathrm{~s}$.

Those looking for dramatic breakthroughs may be a bit disappointed. Neural-network models have not yet solved the problem of how we store memories, nor have they found a cure for schizophrenia. What has happened instead has been a slow, steady fitting of more and more pieces into what Stephen Grossberg-one of the pioneers in neural network theory-has compared to an enormous jigsaw puzzle the size of a hotel ballroom. While there have been multiple, often conflicting, quantitative models of the same psychological phenomena, some broad general principles have emerged. In this article, I will use some of my own work to illustrate methods for employing these principles in modeling.

One of the major problems in psychology is that of selective attention. How does a person or animal select, from the multitude of stimuli it encounters, those that will be predictive of important positive or negative consequences in the future, and learn to act on them appropriately? The solutions to this problem in a neural-network context have broad implications not only for the psychologist, but also for the engineer who seeks to build goal direction or planning into intelligent devices. A collection of articles in progress (Levine \& Leven, 1989) focuses on the interconnected issues of motivation, emotion, and goal direction. Werbos (1988) posed the issue of reinforcement learning: how an organism learns to con-

Address correspondence to Daniel S. Levine, Department of Mathematics, University of Texas at Arlington, Arlington, TX 76019. trol its environment to maximize positive reinforcement and minimize negative reinforcement.

But it is hard to believe that all decisions by humans or other mammals are based on reinforcement learning. What about gamblers, or drug addicts, or people trapped in bad love affairs that they lack the courage to leave? What about rats who get into a vicious cycle when they learn a response to turn off an electric shock and then get shocked for making that very response? Some writers argue that such people or rats are maximizing net positive reinforcement, but the logic of these arguments seems rather tortured. Most of us have heard nagging friends or counselors say, "You could quit overeating (or leave that relationship, or walk away from that dead-end job) if you really wanted to." Such advice, however, is not always accurate. At times, the more rewarding alternative also involves greater risk, and the known alternative yields a lesser but certain satisfaction. At other times, we engage in an activity not because the activity promises to be rewarding, but only because it is novel. So "wanting" in the sense of maximizing expected satisfaction is not our sole reason for doing things.

The work summarized here is contained in two articles that describe simulations of behavioral data in neural networks. One article (Grossberg \& Levine, 1987) uses blocking data from classical conditioning as a prototype of reinforcement learning. The other article (Levine \& Prueitt, 1989) uses cognitive data on frontally damaged primates as a prototype of deviation from reinforcement learning. The deviating data are the result of brain damage, but, I believe, represent exaggerations of effects that occur in apparently normal people and animalseffects in which habit or novelty is a stronger guide to behavior than is current or anticipated reinforcement.

Figure 1 schematizes the well-known blocking paradigm (Kamin, 1968, 1969). First, a neutral stimulus $\left(\mathrm{CS}_{1}\right)$, such as a tone, is presented several times, followed at a given time interval by an unconditioned stimulus (US), such as an electric shock. This pairing occurs until a conditioned response (CR) is established to the $\mathrm{CS}_{1}$. Next, several 


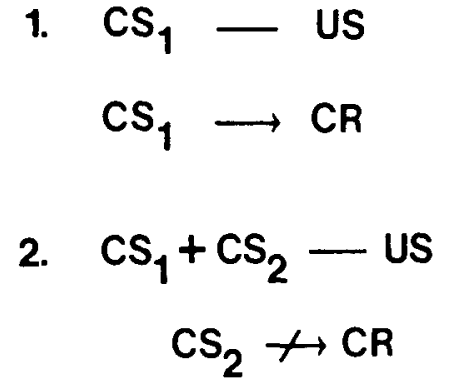

Figure 1. A blocking paradigm. (From "Neural dynamics of attentionally modulated Pavlovian conditioning: Blocking, interstimulus interval, and secondary reinforcement," by $S$. Grossberg and D. S. Levine, 1987, Applied Optics, 26, 5015-5030. Copyright 1987 by the Optical Society of America. Reprinted by permission.)

trials are given in which the $\mathrm{CS}_{1}$ and another neutral stimulus $\left(\mathrm{CS}_{2}\right)$, such as a light, are presented simultaneously, followed at the same time interval by the US. Finally, the $\mathrm{CS}_{2}$ is presented alone but is not reinforced. On these recall trials, no $\mathrm{CR}$ occurs in response to the $\mathrm{CS}_{2}$.

To simulate blocking in a neural network, Grossberg and Levine (1987) broke up the selective-attention problem into four subproblems:

How does the pairing of $\mathrm{CS}_{1}$ with US in the first phase of the blocking experiment endow the $\mathrm{CS}_{1}$ cue with properties of a conditioned, or secondary, reinforcer? How do the reinforcing properties of a cue, whether primary (US) or secondary $\left(\mathrm{CS}_{1}\right)$, shift the focus of attention toward its own processing? How does the limited capacity of attentional resources arise, so that a shift of attention toward one set of cues ... can prevent other cues ... from being attended? How does withdrawal of attention from a cue prevent that cue from entering into new conditioned relationships? (p. 5016)

Once a large cognitive problem is divided into subproblems, the subproblems suggest neural-network design principles that can, in turn, be concatenated to solve other large cognitive problems. In this case, our selectiveattention mechanism combines the principle of associative modification of connection strengths with the principle of competition between sensory representations. Both of these principles will be illuminated below.

The conditioning models simulated in Grossberg and Levine (1987) and in Grossberg and Schmajuk (1987) are part of a general theory developed in Grossberg (1971, 1972a, 1972b, 1975, 1982a, 1982b). This theory relies on a third principle, in addition to association and competition, to account for responses to changes in reinforcement over time. For example, in the blocking paradigm, Kamin $(1968,1969)$ found that if the $\mathrm{CS}_{1}-\mathrm{CS}_{2}$ complex is paired with a different level of shock than $\mathrm{CS}_{1}$ alone, blocking may not occur. Also, it is well known that an instrumental response that terminates an aversive stimulus such as a shock becomes positively reinforced, even though the response is associated with the absence of a negative reinforcer, rather than with the presence of a concrete positive reinforcer. Both of these temporal-contrast effects can be explained by a mechanism known as opponent processing.

The following three sections give capsule descriptions of the general principles of association, competition, and opponency. These principles are frequently combined in various ways to build neural networks, just as resistors, capacitors, and transistors are combined in various ways to build electronic networks. Later sections will discuss how these principles are combined in the networks of Grossberg and Levine (1987) and in those of Levine and Prueitt (1989).

\section{The Principle of Association}

The idea of associative learning goes back at least to Pavlov (1927) and Hull (1943). Hebb (1949) proposed a neuronal basis for such learning:

When the axon of cell $A$ is near enough to excite a cell $B$ and repeatedly or persistently takes part in firing it, some growth process or metabolic change takes place in one or both cells such that A's efficiency, as one of the cells firing $B$, is increased. (p. 64)

Repeated firing of cell B by cell A is a neural correlate of repeated presentation of two stimulus events in sequence, such as a bell followed by food, or the letter A followed by the letter $B$.

Grossberg (1969) incorporated the effects of correlated cell activities on associations into differential equations for a neural network. However, in Grossberg's networks, Hebb's (1949) postulate is not obeyed because the correlation process is counteracted by other processes that tend to decrease associative strength even with repeated pairing. These processes are slow, passive decay of associative strength and competitive inhibition between stimulus traces.

The associative law is embodied in equations that relate two cell population activities $x_{i}$ and $x_{j}$, and the connection strength $z_{i j}$ between them (see Figure 2). The units in such networks are selected populations of neurons rather than single neurons. Their activities are assumed to be deterministic; firing frequencies average the random firing of many thousands of neurons. In this context, $z_{i j}$ is interpreted as an average amount of chemical transmitter at interpopulation synapses. The equations are of the form

$$
\begin{aligned}
& d x_{j} / d t=-a x_{j}+f\left(x_{i}\right) z_{i j} \\
& d z_{i j} / d t=-c z_{i j}+f\left(x_{i}\right) g\left(x_{j}\right),
\end{aligned}
$$

where $a$ and $c$ are decay rates ( $c$ much less than $a$ ), and $f$ and $g$ are "signal functions"' that denote neuronal inputoutput transformations. A variant of this model replaces Equation 2 by

$$
d z_{i j} / d t=\left[-c z_{i j}+g\left(x_{j}\right)\right] f\left(x_{i}\right),
$$

In Equation 2', synaptic strength remains constant in the absence of presynaptic activity, and decays if presynaptic activity occurs without being followed by postsynaptic ac- 


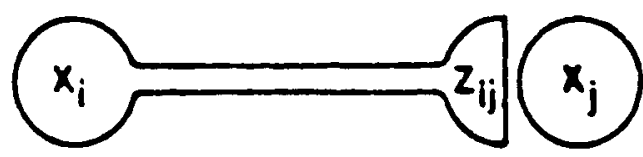

Figure 2. Schematic of two elements and one modifiable synapse between them in the Grossberg model. (From "Neural population modeling and psychology: A review," by D. S. Levine, 1983, Mathematical Biosciences, 66, 1-86. Copyright 1983 by Elsevier Science Publishing Company. Reprinted by permission.)

tivity. Such a mechanism is analogous to associative learning between two stimuli $A$ and $B$, which is stable in the absence of $A$ but decays if $A$ occurs and is not followed by $\mathrm{B}$.

The associative (sometimes known as "Hebbian") law is not the only law of synaptic modification, or learning, used in model neural networks, but it is one of the most common. In addition to many articles by Grossberg and his colleagues, this law also appears in Anderson, Silverstein, Ritz, and Jones (1977), Kohonen (1984), Kohonen et al. (1977), Malsburg (1973), and Rumelhart and Zipser (1985), for example. In addition, there has been a growing body of evidence for an analogous law occurring at the single-neuron level in both invertebrates and mammals; this physiological evidence is summarized in Byrne (1987).

\section{The Principle of Competition}

The selective-attention mechanism developed by Grossberg (1975) depends on previous work (Grossberg, 1973) on perception without learning. His perceptual model addressed the question of how the more significant or intense parts of a pattern (e.g., the brighter areas of a visual pattern) are enhanced, and how the internal noise of cell populations is suppressed.

Both selective attention and contrast enhancement arise from competitive inhibition between cell populations that code different stimuli. Such competition generalizes the lateral inhibition that is common in the visual system (Hartline \& Ratliff, 1957; Kuffler, 1953). In Figure 3, each population excites itself and inhibits the others. A more realistic version of this effect is for each population both to excite and to inhibit all others, but to send excitation over a shorter distance than inhibition.

The equations used by Grossberg (1973) for on-center off-surround networks are of the general form

$$
\begin{aligned}
d x_{i} / d t= & -a x_{i}+\left(B_{i}-x_{i}\right) \sum_{k=1}^{n} f_{k}\left(x_{k}\right) c_{k i} \\
& -x_{i} \sum_{k=1}^{n} f_{k}\left(x_{k}\right) d_{k i}+I_{i}
\end{aligned}
$$

for the $i$ th population's activity. Equation 3 combines three effects: (1) decay back to a baseline level activity, with decay rate $a ;(2)$ excitation from other populations, with interaction coefficients $c_{k i}$; and (3) inhibition from other populations, with interaction coefficients $d_{k i}$. The function $f$ is a "signal function"; the multiplicative (shunting) terms $B_{i}-x_{i}$ and $-x_{i}$ are neurophysiologically justified in Grossberg (1973). Similar equations appear in other competitive neural networks (e.g., Amari, 1977, 1980; Bienenstock, Cooper, \& Munro, 1982, McClelland \& Rumelhart, 1981; Wilson \& Cowan, 1972, 1973).

Cohen and Grossberg (1983), Grossberg (1973, 1978), and Grossberg and Levine (1975) proved theorems about the large-time behavior of Equation 3 and related equations. They showed that if the values of $x_{i}$ at time 0 are interpreted as a sensory-input pattern, and the limiting values of $x_{i}$ as time approaches infinity are interpreted as the stored version of that pattern in short-term memory, then the stored pattern represents some part of the input pattern. Typically, the part of the pattern that is stored depends on the signal function $f$; if $f$ is a properly chosen nonlinear function, the transformed pattern can represent the original pattern with contrast enhanced and noise suppressed. Thus, competition sometimes means that only the most intense inputs survive in short-term memory ("winner take all"), but at other times means that all inputs above a certain threshold intensity are stored in proportion to their original strength.

\section{The Principle of Opponency}

Grossberg (1972a, 1972b) asked how an animal can learn a motor response that terminates a painful stimulus such as a shock. For that to occur, an associative synapse has to be strengthened between representations of the response and of some source of reward. But how is removal of negative reinforcement translated into positive reinforcement? Offset of inhibitory activity at one neural locus must lead to transient onset of excitatory activity at another neural locus.

More generally, a method is needed to compare current values of variables with ongoing values, either stimulus intensity or reinforcement strength. To do so, some researchers (Klopf, 1988; Kosko, 1986) have suggested a differential Hebbian learning rule whereby a synapse is strengthened by the correlation of derivatives (i.e., rates of change) of presynaptic and postsynaptic activities.

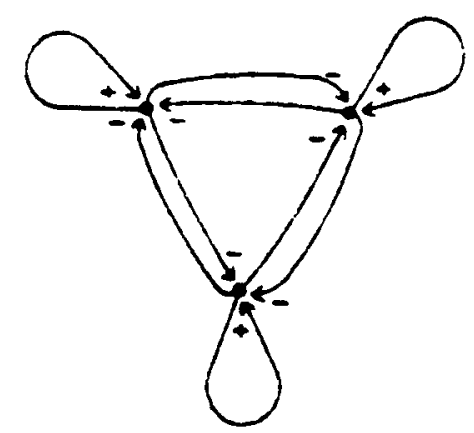

Figure 3. An example of a recurrent lateral-inhibitory network. (From "Neural population modeling and psychology: A review," by D. S. Levine, 1983, Mathematical Biosciences, 66, 1-86. Copyright 1983 by Elsevier Science Publishing Company. Reprinted by permission.) 
Differential Hebbian rules, however, are sensitive to intensity but not to duration of previous stimuli and reinforcements. For this reason, we prefer a different comparison method based on transmitter habituation.

The mechanism proposed by Grossberg (1972a, 1972b) for generating transient rebounds is called opponent processing, or the gated dipole theory. His network, as shown in Figure 4, has two sides ("channels"), one of which has a positive, the other a negative effect on motor input. Grossberg proposed that activity of some synapses not only increases production of a chemical transmitter, via an associative mechanism like that of Equations 1,2 or Equations 1, 2', but also increases the transmitter's release and, therefore, depletion. Ongoing stimulation of one of the two channels counteracts transmitter depletion, making that channel the more active of the two. At the termination of such stimulation, however, transmitter depletion takes over, and since both channels are receiving the same arousal input, the opposite channel becomes transiently more active.

To combine transmitter production and release, one can, for example, take Equations 1 and 2 with $z_{i j}$ replaced in Equation 1 by $Z$, where $Z$ denotes the amount of available transmitter and $z$ denotes the rate of transmitter produc-

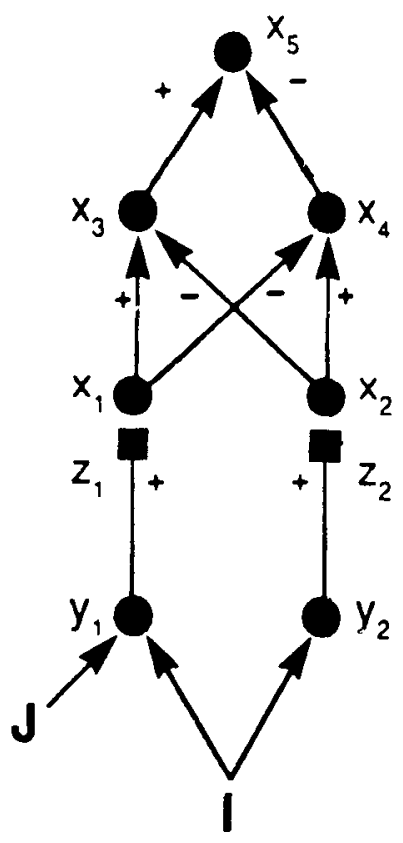

Figure 4. Schematic of a gated dipole with two competing channels ("fear and relieP" channels if the input $J$ is electric shock; "on and of " channels if $J$ is a given sensory stimulus). I is a nonspecific arousal to both channels. Synapses with depletable transmitters are denoted by filled-in squares. While $J$ is on, the left channel's greater input overcomes that channel's greater transmitter depletion; hence $x_{3}>x_{4}$, and $x_{5}$ is excited. After $J$ is turned off, the greater depletion of the left channel, combined with equal channel inputs $I$, causes rebound activity in the right channel; hence, transiently, $x_{4}>x_{3}$, and $x_{5}$ is inhibited. (From "Modeling some effects of frontal-lobe damage: Novelty and perseveration," by D. S. Levine and P. S. Prueitt, 1989, Neural Networks, Vol. 2, March. Copyright 1989 by Pergamon Press. Reprinted by permission.) tion. $Z$ is, in turn, described by an equation of the form

$$
d Z / d t=h(k z-Z)-r x Z .
$$

The three terms in Equation 4 describe, respectively, transmitter production rate proportional to $z$, feedback inhibition proportional to the amount of transmitter, and transmitter release proportional to the amount of transmitter and to presynaptic activity.

The theory of reinforcement in a gated dipole was quantified by Grossberg (1972b). Such opponent processing could explain not only positive reinforcement from termination of an aversive stimulus, but also negative reinforcement (frustration) from either termination of a rewarding stimulus or absence of an expected reward. In addition, Grossberg (1980) generalized the gated-dipole idea from the reinforcement to the sensory domain, with "on channels" and "off channels" for given sensory stimuli. This suggests a model for enhancement of the effects of novel stimuli, a topic to which we will return below. Another neural network with a similar approach to novelty detection was developed in Kohonen (1984) and Kohonen and Oja (1976).

\section{Simulation of Attentional Effects}

Many neural network models of classical conditioning (e.g., Klopf, 1988; Sutton \& Barto, 1981) use some form of modifiable synapse between the sensory representations for the CS and the US. Grossberg (1971), however, showed that this could cause difficulties if more than one sensory event occurred in a short time-interval. In his previous work, each elementary sensory representation had been interpreted mathematically as a pattern of activation across many cell populations. If a US was interspersed with one or more other pattern(s), the CS population could then become associated, not to the US, but to a noisy mixture of the US and the other impinging stimuli.

To prevent losing the US in the confusion of other events, Grossberg (1971) proposed the existence of populations of cells that are separate from sensory representations of particular stimuli but related to particular drives and emotions. These "drive nodes" are not drive-reduction areas, but are areas analogous to loci in emotional regions of the brain-such as the hypothalamus-where mechanisms of conditioning, reinforcement, homeostasis, and competition interact (see Grossberg, 1982a, 1982b, for more discussion). Each US has a sensory representation, called $S_{U S}$, which is strongly connected to some drive node; for example, a food US unconditionally activates the hunger drive node $\left(D_{H}\right)$ if the hunger level is sufficiently high. The distinction between $S_{U S}$ and $D_{H}$ mirrors a distinction drawn by Hebb (1955) between two separate functions for each sensory event: its cue function, which guides behavior, and its arousal function, which energizes behavior.

Hence, repeated pairing of a neutral CS with a food US causes pairing of stimulation of the CS sensory representation $\left(S_{C S}\right)$ with the hunger-drive representation $D_{H}$. If the $S_{C S}$-to- $D_{H}$ synapses are assumed to be modifiable according to an associative rule (see the Principle of As- 
sociation section, above), the pairing can become strengthened so that eventually the CS by itself activates the drive representation directly.

Figure 5 shows the network used in the simulations by Grossberg and Levine (1987). The synapses denoted by semicircles are subject to modification according to an associative rule. These associative synapses are between sensory $(S)$ nodes and drive $(D)$ nodes. Learning at $S$-to$D$ synapses is called conditioned-reinforcer learning because it enables a previously neutral stimulus to become a secondary reinforcer after it is conditioned to a US. Learning at $D$-to- $S$ synapses is called incentivemotivational learning because it yields incentives to approach or to avoid given CSs based on their reinforcement value. Selective attention occurs at modifiable $D$-to- $S$ synapses, mediated by competition for short-term memory between $S$ nodes. There are three $S$ representations, corresponding to two conditioned stimuli $\left(\mathrm{CS}_{1}\right.$ and $\left.\mathrm{CS}_{2}\right)$, and to one unconditioned stimulus (US). Each sensory representation is broken up into two stages $\left(x_{i 1}\right.$ and $\left.x_{i 2}\right)$ for technical reasons beyond the scope of this article.

Simulations of a blocking paradigm that use the above network are shown in Figure 6. First, the $\mathrm{CS}_{1}$ is presented five times, followed by the US at a time interval ( 6 timeunits) that is conducive to rapid learning with the given parameter values. Then the $\mathrm{CS}_{1}-\mathrm{CS}_{2}$ pair is presented five times, followed by the US at the same time interval. As Figures $6 c$ and $6 d$ show, the $\mathrm{CS}_{2}$ has not become appreciably conditioned to the US, in spite of having been presented often enough to have been so conditioned in the absence of $C S_{1}$. This is a clean demonstration of selective attention and reinforcement learning in a model neural network.

\section{Simulation of Novelty and Habit Effects}

As the above section shows, selective attention to reinforcing stimuli depends on feedback between sensory and drive representations. The frontal cortex appears to be a part of such feedback, since it is the area of the cerebral cortex that has the strongest connections with the limbic system, and is also the only cortical area known to have direct connections with the hypothalamus (Nauta, 1971).

Hence, frontal damage leads to characteristic defects in guidance and planning of behavior. Some of these defects are mutually paradoxical: frontal lesions can lead both to perseveration in formerly (but no longer) rewarding behavior (Milner, 1963, 1964; Spaet \& Harlow, 1943) and to excessive preference for novel stimuli (Pribram, 1961). Yet Nauta (1971) saw both of these defects as part of the diminished influence of present or anticipated reinforcement on behavior through the severing of links between the cortical sensory and motor areas and the sub-

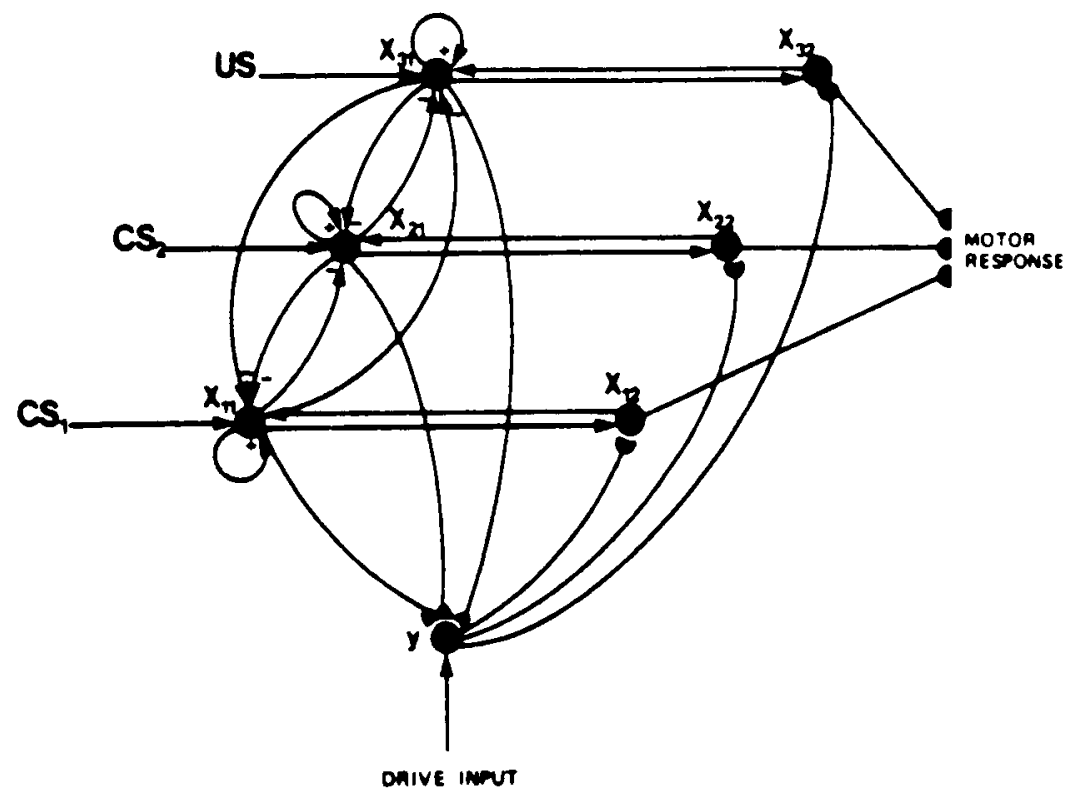

Figure 5. A network used in blocking simulations. Each sensory representation has two stages with short-term memory activities $x_{11}$ and $x_{i 2}$. A CS or US activates its corresponding $x_{i 1}$. Activation of $x_{11}$ generates unmodifiable signals to $x_{12}$ and conditioned reinforcer signals to $D$, whose activity is denoted by $y$. Incentive motivational feedback from $D$ activates the second stage $x_{n}$, which sends feedback signals to $x_{i 1}$. Motor learning is elicited by signals from $x_{n}$ to motor-command representations. Long-term memory traces are denoted by semicircles. (From "Neural dynamics of attentionally modulated Pavlovian conditioning: Blocking, interstimulus interval, and secondary reinforcement," by S. Grossberg and D. S. Levine, 1987, Applied Optics, 26, 5015-5030. Copyright 1987 by the Optical Society of America. Reprinted by permission.) 

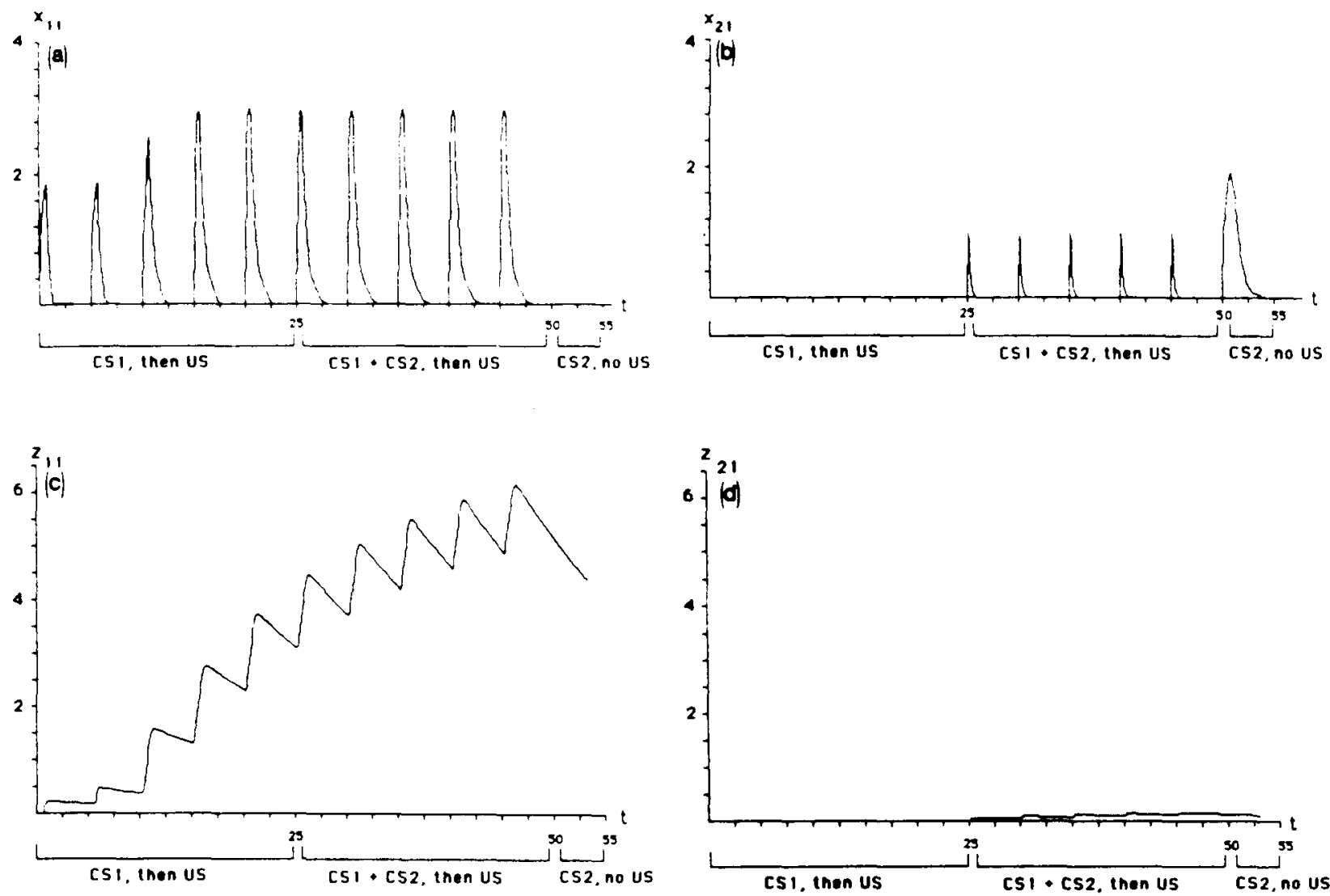

Figure 6. Blocking simulation. Five trials of $\mathrm{CS}_{1}-\mathrm{US}$ pairing are followed by five trials of $\mathrm{CS}_{1}+\mathrm{CS}_{2}-\mathrm{US}$ pairing. Then $\mathrm{CS}_{2}$ is presented alone for one trial. (a) Activity $x_{11}$ of $S_{c s_{1}}$ through time; (b) Activity $x_{21}$ of $S_{c s_{2}}$ through time; (c) $S_{c s_{1}}$-to-D connection strength $z_{11}$ through time; (d) $S_{C s_{1}}$-to-D connection strength $z_{21}$ through time. (From "Neural dynamics of attentionally modulated Pavlovian conditioning: Blocking, interstimulus interval, and secondary reinforcement," by S. Grossberg and D. S. Levine, 1987, Applied Optics, 26, 5015-5030. Copyright 1987 by the Optical Society of America. Reprinted by permission.)

cortical motivational areas. The networks in our laboratory (Levine and Prueitt, 1989) were built on Nauta's qualitative analysis.

We sought to illuminate habit and novelty effects by constructing networks in which one or both of these types of effects dominate decisions when reinforcement signals are weakened (Levine \& Prueitt, 1989). Such a weakening of reinforcement is used to mimic frontal damage, and was done by simulation of two typical frontal-damage effects: (1) perseveration in frontally damaged human patients taking a sorting test (Milner, 1963, 1964), and (2) novelty preference in frontally lesioned monkeys choosing between objects (Pribram, 1961). Future articles will expand on this work, with the ultimate goal of building a computational "wiring diagram" of the frontal lobes and their connections with other brain areas.

Milner $(1963,1964)$ compared brain-damaged and normal subjects on the Wisconsin card-sorting test. In those studies, a subject was given a sequence of cards, each of which showed a number, color, and shape. The subject's task was to match each card to one of four template cards (see Figure 7). The experimenter would indicate "Right" or "Wrong," but would give no reason. After 10 consecutive correct matches to color, the criterion was changed to shape without warning. If 10 consecutive correct matches were made to shape, the criterion was shifted to number, then back to color, and so on. Most patients with damage to the dorsolateral frontal cortex learned the color criterion as quickly as did the normals, but could never switch to shape. In contrast, most normals and patients with other brain lesions achieved 10 consecutive correct responses four or five times over 128 trials.

Milner's $(1963,1964)$ results were simulated using the neural network of Figure 8, which builds on the adaptiveresonance network of Carpenter and Grossberg (1987) (ART 1). ART 1 was designed to classify input patterns, with one field of nodes coding for input features and another field of nodes coding for categories. Levine and Prueitt (1989) added a mechanism for attentional bias within the feature field to the categorization network (Grossberg \& Levine, 1975).

In the network of Figure 8, nodes in $F_{1}$ code for features (numbers, colors, shapes). Nodes in $\mathrm{F}_{2}$ code for template cards, each of which creates a category of cards simi- 
TEMPLATE CARDS
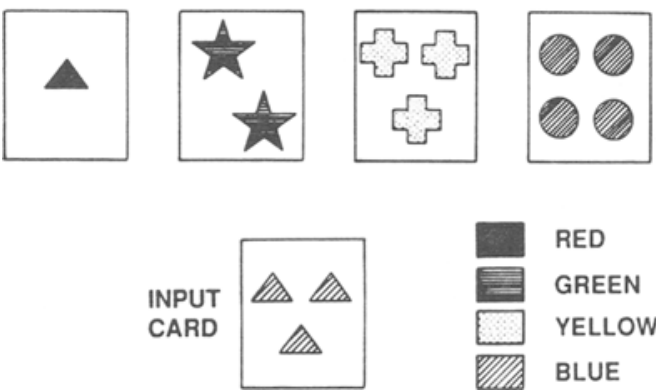

RED

GREEN

YELLOW

BLUE

Figure 7. Cards used in the Wisconsin card-sorting test. (From Modeling some effects of frontal-lobe damage: Novelty and perseveration," by D. S. Levine and P. S. Prueitt, 1989, Neural Networks, Vol. 2, March. Copyright 1989 by Pergamon Press. Reprinted by permission.) lar to it. $F_{1}$ has three subfields (number, color, and shape). To each subfield there corresponds a "habit node" and a "bias node," apart from $F_{1}$ and $F_{2}$. Habit nodes detect how often a criterion has been used, rightly or wrongly, for sorting. Bias nodes respond both to reinforcement signals (here, the statement of "Right" or "Wrong") and to habit-node signals.

Interfield synapses are strong between nodes for a given feature and category nodes for cards that include that feature. These synapses are selectively enhanced by attentional gating from bias nodes; for example, if color bias is higher than shape bias, the "one red triangle" node at $F_{2}$ will be more excited by the "red" node than by the "triangle" node at $F_{1}$. When an input card is seen, the template card with the largest activity in response to the input will be chosen as the one matched. If the card chosen and the input card match in color, shape, or number, a

\section{CATEGORIES $\left(\mathrm{F}_{2}\right)$}

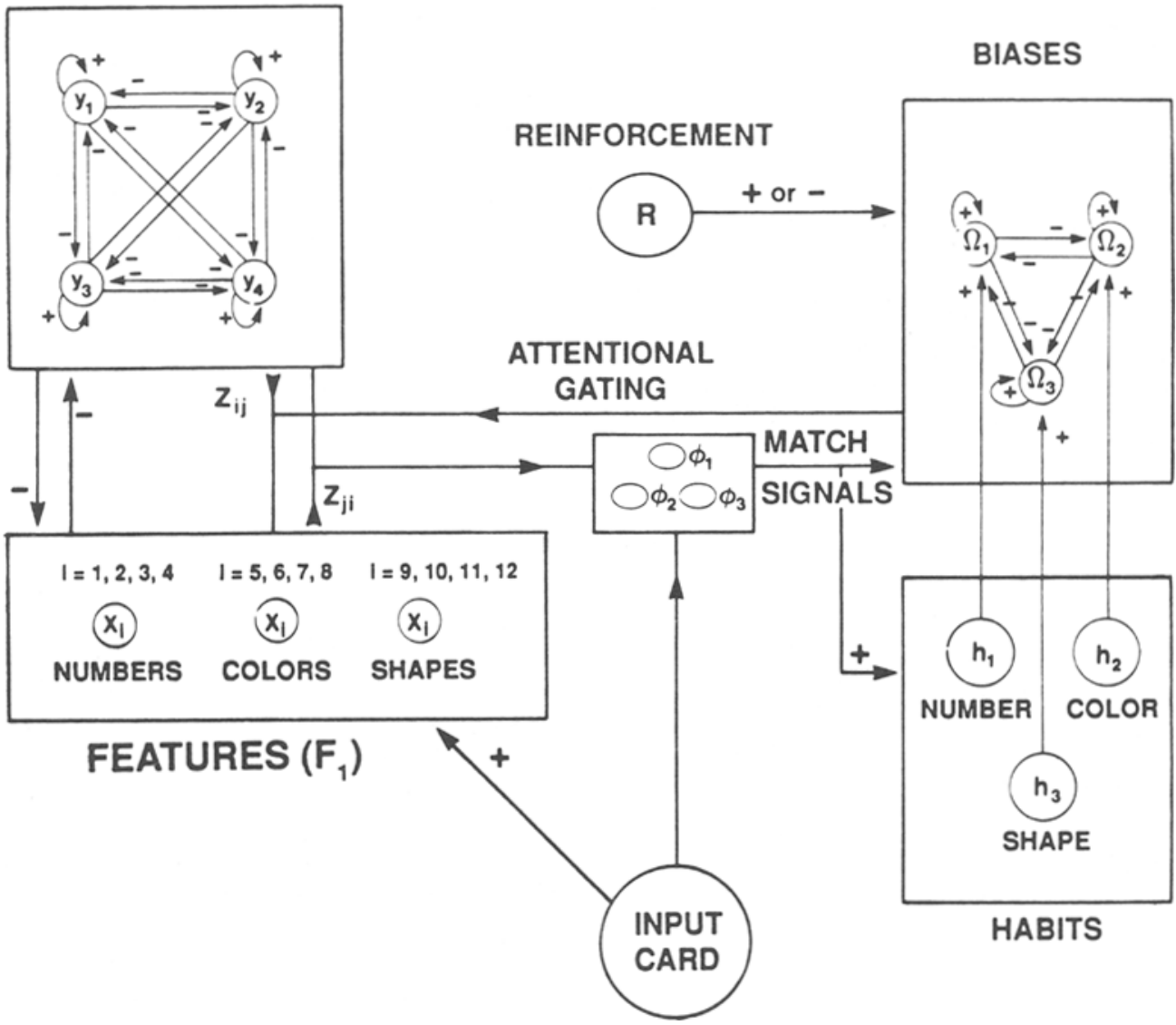

Figure 8. The neural network used to simulate card-sorting data. Frontal-lobe damage is modeled by reduced gain of positive and negative signals from reinforcement node $R$ to bias nodes $Q_{t}(i=1$ for number, 2 for color, 3 for shape). Each bias node also receives a signal from the corresponding habit node, which encodes past decisions based on the given criterion. The match signal generators $\Phi_{1}$ send positive signals to habit nodes, and signals to bias nodes with the same sign as the reinforcement. Bias nodes in turn gate signals from feature to category nodes. (Modified from “Effects of reinforcement on knowledge retrieval and evaluation," by S. J. Levin and D. S. Levine, 1987, Proceedings of the IEEE First International Conference on Neural Networks, Volume II, pp. 269-279. Copyright 1987 by IEEE. Reprinted by permission.) 
"'match signal" goes to the corresponding habit and bias nodes. This signal increases or decreases the bias node's activity, depending on whether or not the choice was correct. Inhibition from $F_{1}$ to $F_{2}$ lets $F_{2}$ reset to new inputs; inhibition from $F_{2}$ to $F_{1}$ prevents top-down signals from being seen as inputs.

Separation of habit and reinforcement loci is supported by behavioral studies on macaque monkeys (Mishkin, Malamut, \& Bachevalier, 1984). Mishkin et al. found evidence from lesion data that memories of motor responses and memories of the reinforcement values of events are encoded in interacting but separate neural systems. The motor-habit system centers in the basal ganglia, and the reinforcement system centers in the hippocampus and amygdala.

Our model uses the fact that the frontal lobes are the part of the cortex most directly connected with subcortical motivational areas (Nauta, 1971). However, other coriical regions connect with these areas less directly. Therefore, when simulating frontal damage, we set $\alpha$ to be small but not zero. If reinforcement gain were zero, sorting by color could not be learned at all. With weak reinforcement, choice patterns based on reward can be learned if no competing habits exist. Once a choice pattern has become a habit, though, it perseverates because reward changes have too little influence to overcome the habit.

Figure 9 shows the results of our simulations. The parameter $\alpha$ measures gain of signals (positive or negative) from the reinforcement node to bias nodes. With high $\alpha$, the network acted like one of Milner's normal subjects, making 10 consecutive correct responses five times during 128 trials. With low $\alpha$, the network acted like one of Milner's dorsal-frontal patients; it learned the color criterion as rapidly as did the "normal" network, but it classified on the basis of color for all remaining trials.

As for novelty, Pribram (1961) compared normal and frontally lesioned rhesus monkeys in a scene with many different junk objects. First, a monkey was presented with one cue, a junk object placed over 1 of 12 holes drilled in a board. A peanut had been placed under this cue. After the monkey lifted the cue a fixed number of times and

$\begin{array}{llr} & \text { CRITERION } & \text { TRIAL } \\ \alpha=4 & \text { Color } & 13 \\ \text { ("Normal") } & \text { Shape } & 40 \\ & \text { Number } & 82 \\ & \text { Color (again) } & 96 \\ & \text { Shape (again) } & 115 \\ \alpha=1.5 & \text { Color } & 13 \\ \text { ("Frontally } & \text { Thereafter, classified by color } \\ \text { Damaged") } & \text { for all remaining trials }\end{array}$

Figure 9. Results of simulations on the network of Figure 8. Trial number is the first one on which the network achieved 10 consecutive correct matches based on the given criterion. (From "Modeling some effects of frontal-lobe damage: Novelty and perseveration," by D. S. Levine and P. S. Prueitt, 1989, Neural Networks, Vol. 2, March. Copyright 1989 by Pergamon Press. Reprinted by permission.)

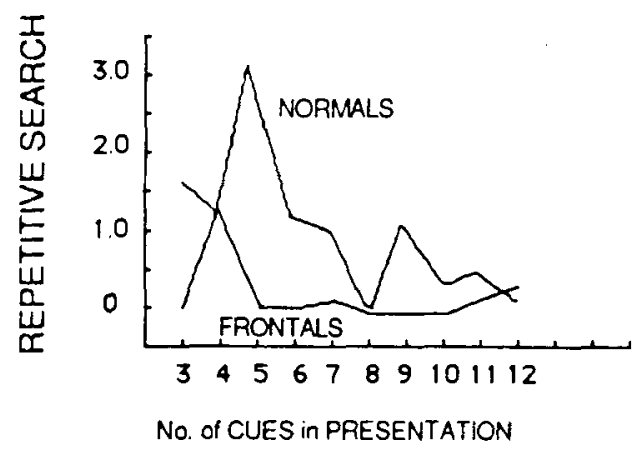

Figure 10. Experimental data on frontally damaged versus normal rhesus monkeys in a scene with up to 12 different junk objects. A reward was placed under the novel object each time. The graph shows the mean number of times the monkey approached the previously rewarded object before reaching a set number of consecutive responses to the novel object. (Modified from "A further experimental analysis of the behavioral deficit that follows injury to the primate frontal cortex," by K. Pribram, 1961, Journal of Experimental Neurology, 3, 432-466. Copyright 1961 by Academic Press. Reprinted by permission.)

was rewarded, a second (novel) junk object was added and placed over another of the 12 holes while the board was hidden from the monkey's view. Again, the reward was placed under the novel cue. This process was repeated until all holes were covered with junk objects. Each time, the peanut remained under the same (novel) cue until the animal found the reward a fixed number of times.

Figure 10 shows Pribram's data. The number of repetitive errors (lifting the familiar object) before the monkey first selected the novel object is shown for both normal and frontally damaged ("frontal") subjects. In general, frontal animals, which were more attracted to the novel object, made fewer errors than did normals.

Recall from the Principle of Opponency section above that gated dipoles provide a method for comparing current values of variables with ongoing values, either stimulus intensity or reinforcement strength. In the sensory domain, a gated dipole consists of "on channels" and "off channels" that respond to the presence or absence of a particular sensory stimulus (Grossberg, 1980). On and off channels for many different stimuli are joined into a "dipole field."

Figure 11 shows a dipole field used to model Pribram's data. Two dipole channel pairs, which correspond to an old cue and a novel cue, are shown. The nodes $x_{1,5}$ and $x_{2,5}$ of the dipoles represent tendencies to approach the given cues. Inhibitory links between these nodes, and a node $x_{3,5}$ that codes some other cue in the environment, denote the competition between these tendencies. The cue with largest $x_{1,5}$ at a given time is approached. The reward node is excited when the monkey finds the peanut. Each $x_{i, 5}$ connects with the reward node via modifiable synapses. Hence, competition is biased in favor of previously rewarded objects. It is also biased in favor of novel objects because their on channels have undergone less transmitter depletion than have those for old cues. 
Figure 12 shows the results of simulating the above dipole field. As the graph shows, with large gain-of-reward signals, the effect of the previous reward was stronger than the effect of novelty, so the previously rewarded cue was approached. With small reinforcement gain, the novel cue was approached. The same qualitative result was obtained in dipole fields with up to 12 dipoles.

The extreme ends of the graph in Figure 9 show that performance of frontal animals deteriorates for small numbers of cues, and performance of normal animals increases for large numbers of cues. We suggest refinements of the above network to account for these effects, which are not given here for space reasons (Levine \& Prueitt, 1989).

\section{Discussion: The Parable of the}

Watchmakers Revisited

There are now several thousand active neural-network researchers, and their network architectures vary widely (see Levine, 1983, for a review, to be updated in Levine,
1989). This variety of networks reflects the diversity of ways in which any given cognitive function can be organized across many biological species and among individuals within these species. In spite of this diversity, however, some subnetwork-organizing principles occur repeatedly in network models that arise from many sources. These principles are ignored at the peril of the modeler. Builders of psychological models or adaptive machines should recall the parable of the watchmakers (Simon, 1969, pp. 90-93). One watchmaker tried to fashion a whole watch simply from fitting the parts together. Another watchmaker started with the same parts but put some of them together into subsystems. Not until the subsystems were working did he join them into a watch. The second watchmaker prospered, whereas the first had to start all over again when interrupted.

In artificial intelligence circles, connectionism has been a recent buzzword. Connectionism is a general term for the use of models, such as those discussed here, based

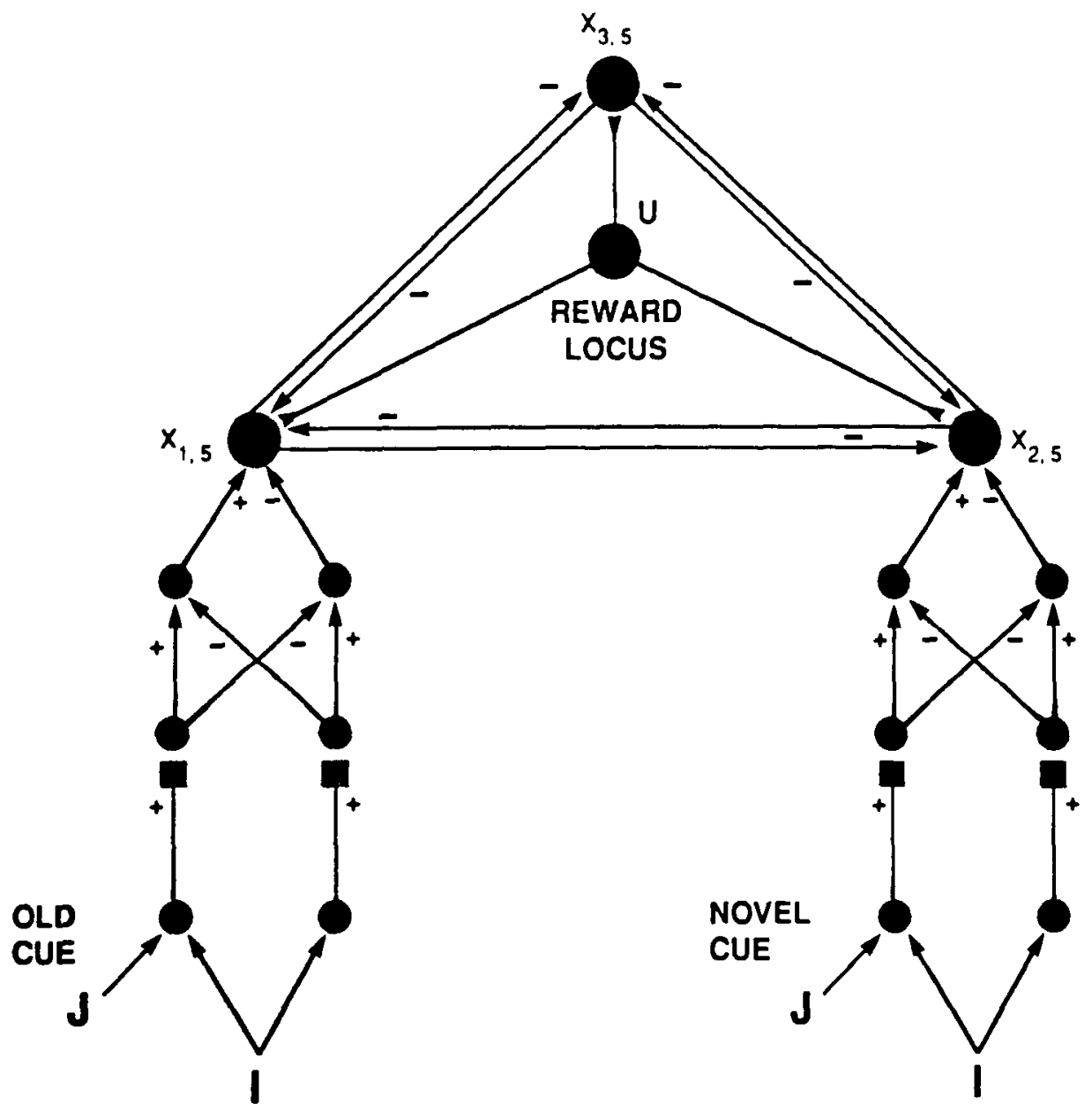

Figure 11. A dipole field, with two of the gated dipoles shown, used to model Pribram's data. One dipole corresponds to an old cue and one to a novel cue. Dipole outputs $x_{i, s}$ represent competing tendencies to approach given cues. Competition among $x_{i, 5}$ nodes is biased by (1) modifiable connections with $u$, giving an advantage to previously rewarded cues, and (2) transmitter depletion at "square" synapses within dipoles, giving an advantage to novel cues. (From "Modeling some effects of frontal-lobe damage: Novelty and perseveration," by D. S. Levine and P. S. Prueitt, 1989, Neural Networks, Vol. 2, March. Copyright 1989 by Pergamon Press. Reprinted by permission.) 
(a)
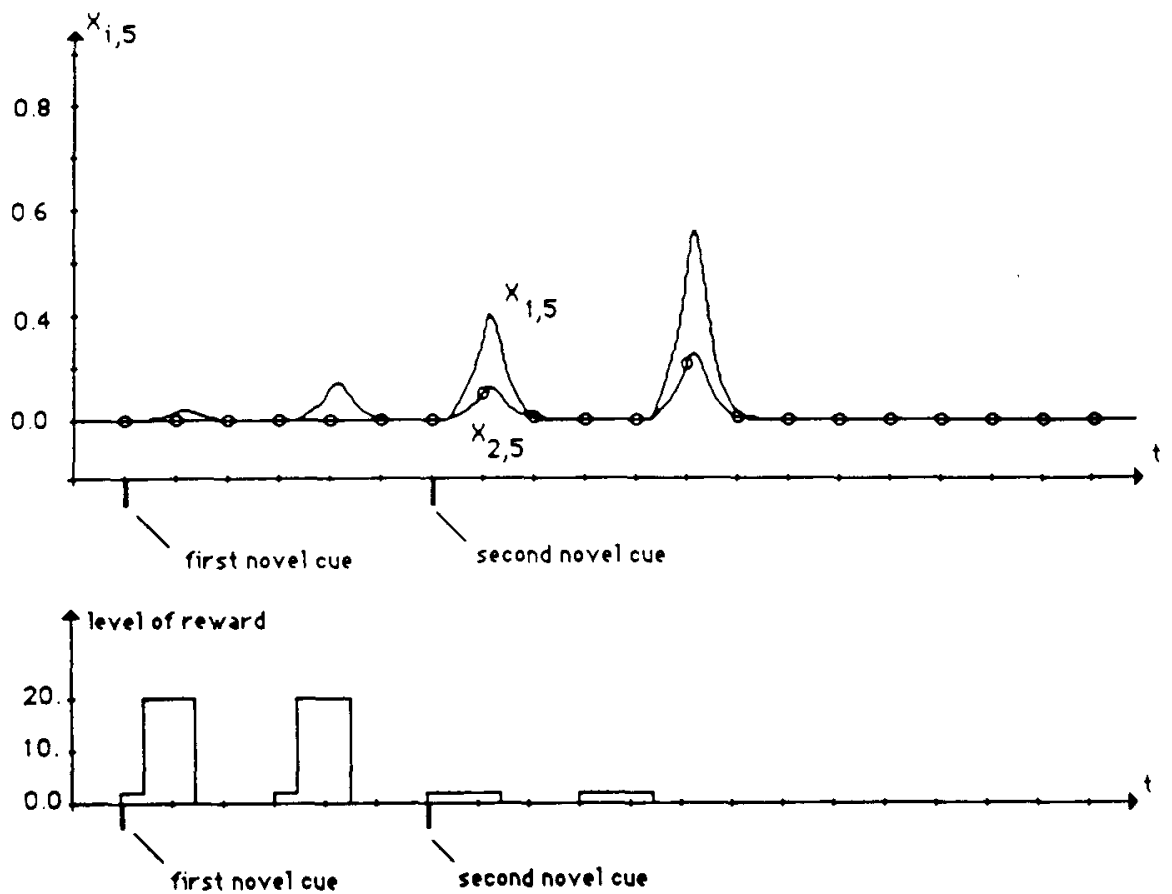

(b)
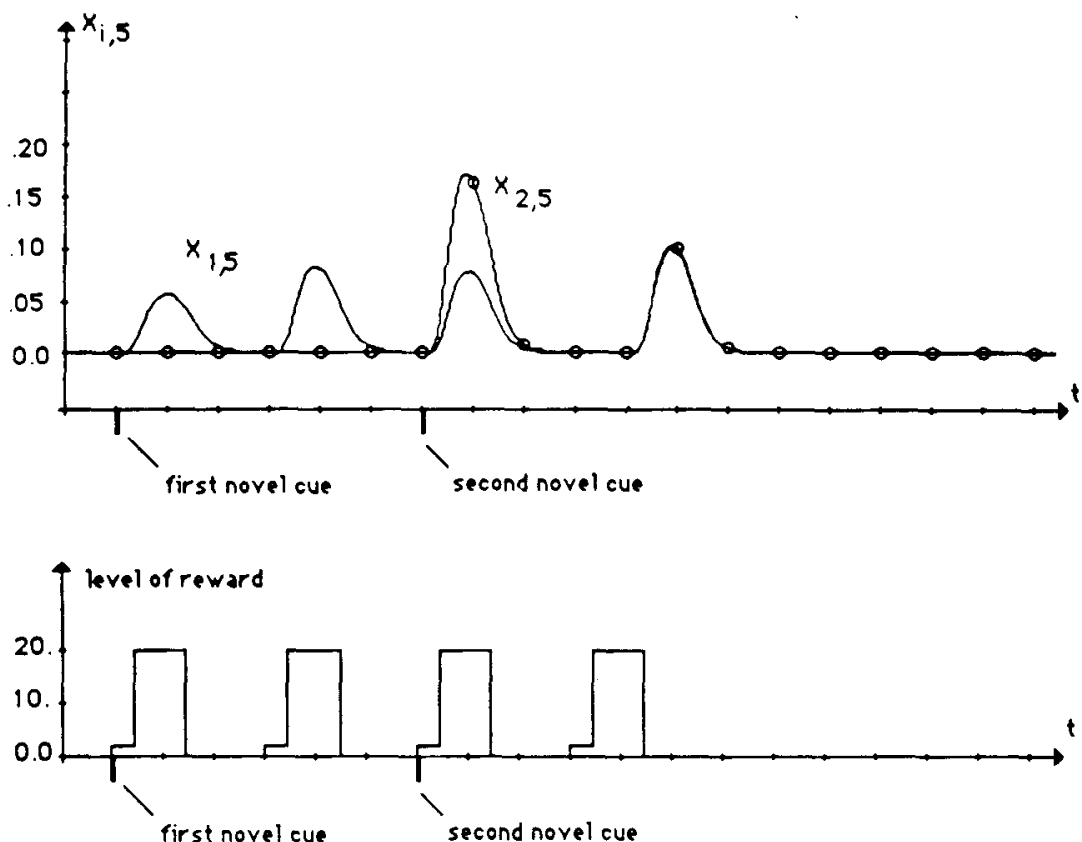

Figure 12. Results of simulations on the network of Figure 11. $x_{1, s}$ denotes an approach to the first cue presented; $x_{2,5}$ denotes an approach to the second cue presented. Dark vertical tick marks show the times of first presentation of novel cues. The bottom graph denotes the value of the reward node activity $u$. (a) In the "normal monkey" network with a large value (2) for gain of reward signals, $x_{1,5}>x_{2,5}$ when both cues are present; so the previously rewarded cue is chosen. (b) In the "frontal monkey" network with a small value (.1) of reward gain, $x_{2,5}>x_{1,5}$ when both cues are present; so the novel cue is chosen. (From "Modeling some effects of frontal-lobe damage: Novelty and perseveration," by D. S. Levine and P. S. Prueitt, 1989, Neural Networks, Vol. 2, March. Copyright 1989 by Pergamon Press. Reprinted by permission.) 
on node activities, internode connection strengths, and rules (usually defined by difference or differential equations) for changes over time of these activities and connection strengths. The connectionist approach is often contrasted with previous approaches based on serial computer programs with intricate instructions but no nodes. It is not informative, however, to state that a model is connectionist without also describing the structure of its connections and some of its useful subsystems.

The principles of association, competition, and opponency, as well as other principles involved in interlevel connections for higher functions, were suggested by a heterogeneous database that is partly physiological and partly psychological. In some cases, such as that of associativelearning laws, the theory was first suggested by psychological results and later verified (at least qualitatively) by physiological results. The interdisciplinary nature of the database lends credence to the ubiquity of these organizing principles in nature.

\section{REFERENCES}

AMARI, S. (1977). Neural theory of association and concept-formation. Biological Cybernetics, 27, 77-87.

AMARI, S. (1980). Topographic organization of nerve fields. Bulletin of Mathematical Biology, 42, 339-364.

Anderson, J. A., Silverstein, J. W., Ritz, S. A., \& Jones, R. S. (1977). Distinctive features, categorical perception and probability learning: Some applications of a neural model. Psychological Review, 84, 413-451.

Bienenstock, E. L., Cooper, L. N., \& Munro, P. W. (1982). Theory for the development of neuron specificity: Orientation specificity and binocular interaction in visual cortex. Joumal of Neuroscience, 2, 32-48.

Byrne, J. H. (1987). Cellular analysis of associative learning. Physiological Reviews, 67, 329-439.

CARPEnTER, G. A., \& Grossberg, S. (1987). A massively parallel architecture for a self-organizing neural pattern recognition machine. Computer Vision, Graphics, \& Image Processing, 37, 54-115.

COHEN, M. A., \& GRossBerg, S. (1983). Absolute stability of global pattern formation and parallel memory storage by competitive neural networks. IEEE Transactions on Systems, Man \& Cybernetics, 13, 815-826.

GrossBerg, S. (1969). Embedding fields: A theory of learning with physiological implications. Journal of Mathematical Psychology, 6, 209-239.

GrossBerg, S. (1971). On the dynamics of operant conditioning. Joumal of Theoretical Biology, 33, 225-255.

Grossberg, S. (1972a). A neural theory of punishment and avoidance. I. Qualitative theory. Mathematical Biosciences, 15, 39-67.

GrossberG, S. (1972b). A neural theory of punishment and avoidance. II. Quantitative theory. Mathematical Biosciences, 15, 253-285.

Grossberg, S. (1973). Contour enhancement, short-term memory, and constancies in reverberating neural networks. Studies in Applied Mathematics, 52, 213-257.

Grossberg, S. (1975). A neural model of attention, reinforcement, and discrimination learning. International Review of Neurobiology, 18, 263-327.

GrossBerg, S. (1978). Competition, decision, and consensus. Journal of Mathematical Analysis \& Applications, 66, 470-493.

Grossberg, S. (1980). How does a brain build a cognitive code? Psychological Review, 87, 1-51.

Grossberg, S. (1982a). Processing of expected and unexpected events during conditioning and attention: A psychophysiological theory. Psychological Review, 89, 529-572.
GrossberG, S. (1982b). A psychophysiological theory of reinforcement, drive, motivation, and attention. Joumal of Theoretical Neurobiology, 1, 286-369.

GrossberG, S., \& LeviNE, D. S. (1975). Some developmental and attentional biases in the contrast enhancement and short-term memory of recurrent neural networks. Journal of Theoretical Biology, 53, 341-380.

Grossberg, S., \& Levine, D. S. (1987). Neural dynamics of attentionally modulated Pavlovian conditioning: Blocking, interstimulus interval, and secondary reinforcement. Applied Optics, 26, 5015-5030.

Grossberg, S., \& Schmajuk, N. (1987). Neural dynamics of attentionally modulated Pavlovian conditioning: Conditioned reinforcement, inhibition, and opponent processing. Psychobiology, 15, 195-240.

HaRTUNe, H. K., \& RATUFF, F. (1957). Inhibitory interaction of receptor units in the eye of Limulus. Journal of General Physiology, 40 , 351-376.

Hebs, D. O. (1949). The organization of behavior. New York: Wiley. HEBB, D. O. (1955). Drives and the CNS (conceptual nervous system). Psychological Review, 62, 243-254.

Hull, C. L. (1943). Principles of behavior. New York: Appleton.

KAMIN, L. J. (1968). Attention-like processes in classical conditioning. In M. R. Jones (Ed.), Miami symposium on the prediction of be havior: Aversive stimulation (pp. 9-31). Miami, FL: University of Miami Press.

Kamin, L. J. (1969). Predictability, surprise, attention, and conditioning. In B. A. Campbell \& R. M. Church (Eds.), Punishment and Aversive Behavior (pp. 279-296). New York: Appleton-Century-Crofts.

KLOPF, A. H. (1988). A neuronal model of classical conditioning. Psychobiology, 16, 85-125.

KoHONEN, T. (1984). Self-organization and associative memory. Berlin: Springer-Verlag.

Kohonen, T., Lehtio, P., Rovamo, J., Hyvarinen, J., Bry, K., \& VAINIO, L. (1977). A principle of neural associative memory. Neuroscience, 2, 1065-1076.

KoHonen, T., \& OJA, E. (1976). Fast adaptive formation of orthogonalizing filters and associative memory in recurrent networks of neuron-like elements. Biological Cybernetics, 21, 85-95.

Kosko, B. (1986). Differential Hebbian learning. In J. S. Denker (Ed.), Neural networks for computing (pp. 265-270). AIP Conference Proceedings Vol. 151. New York: American Institute of Physics.

KUFFLER, S. (1953). Discharge patterns and functional organization of mammalian retina. Journal of Neurophysiology, 16, 37-68.

LeVEN, S. J., \& LEVINE, D. S. (1987). Effects of reinforcement on knowledge retrieval and evaluation. Proceedings of the IEEE First International Conference on Neural Networks (Vol. 2, pp. 269-279). San Diego, CA: IEEE/ICNN.

LeviNe, D. S. (1983). Neural population modeling and psychology: A review. Mathematical Biosciences, 66, 1-86.

LEVINE, D. S. (1989). Introduction to neural and cognitive modeling. Hillsdale, NJ: Erlbaum.

LEVINE, D. S., \& LEVEN, S. J. (Eds.) (1989). Motivation, emotion, and goal direction in neural networks. Hillsdale, NJ: Erlbaum.

Levine, D. S., \& PrueitT, P. S. (1989, March). Modeling some effects of frontal-lobe damage: Novelty and perseveration. Neural Networks, Vol. 2.

MALSBURG, C. VON DER (1973). Self-organization of orientation sensitive cells in the striate cortex. Kybemetik, 14, 85-100.

MCClelland, J. L., \& Rumelhart, D. E. (1981). An interactive activation model of context effects in letter perception: Part 1. An account of basic findings. Psychological Review, 88, 375-407.

MiLNER, B. (1963). Effects of different brain lesions in card sorting. Archives of Neurology, 9, 90-100.

Milner, B. (1964). Some effects of frontal lobectomy in man. In J. M. Warren \& K. Akert (Eds.), The frontal granular cortex and behavior (pp. 313-334). New York: McGraw-Hill.

Mishkin, M., Malamut, B., \& Bachevalier, J. (1984). Memories and habits: Two neural systems. In G. Lynch, J. L. McGaugh, \& N. M. Weinberger (Eds.), Neurobiology of learming and memory (pp. 65-77). New York: Guilford. 
Nauta, W. J. H. (1971). The problem of the frontal lobe: A reinterpretation. Journal of Psychiatric Research, 8, 167-187.

Pavlov, I. P. (1927). Conditioned reflexes (V. Anrep, trans.). London: Oxford University Press.

Pribram, K. (1961). A further experimental analysis of the behavioral deficit that follows injury to the primate frontal cortex. Journal of Experimental Neurology, 3, 432-466.

Rumelhart, D. E., \& Zipser, D. (1985). Feature discovery by competitive learning. Cognitive Science, 9, 75-112.

Simon, H. A. (1969). The sciences of the artificial. Cambridge, MA: MIT Press.

Spaet, T., \& Harlow, H. F. (1943). Problem solution by monkeys following bilateral removal of the prefrontal areas. II. Delayed reaction problems involving use of the matching-to-sample method. Journal of Experimental Psychology, 32, 424-434.
STIPP, D. (1988, September 29). Computer researchers find "neural networks" help mimic the brain. The Wall Street Journal, pp. 1, 22.

SutTon, R. S., \& BARTo, A. G. (1981). Toward a modern theory of adaptive networks: Expectation and prediction. Psychological Review, 88, $135-169$.

Werbos, P. J. (1988). Backpropagation: Past and future. Proceedings of the IEEE International Conference on Neural Networks, 1988 (pp. 343-353). San Diego, CA: IEEE.

WILSON, H. R., \& CowAN, J. D. (1972). Excitatory and inhibitory interactions in localized populations of model neurons. Biophysical Journal, 12, 1-24.

WiLson, H. R., CowaN, J. D. (1973). A mathematical theory of the functional dynamics of cortical and thalamic nervous tissue. Kybernetik, $13,55-80$. 